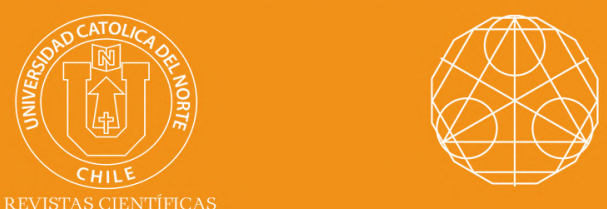

\title{
Oscillation of solutions to a generalized forced nonlinear conformable fractional differential equation
}

\author{
A. Ogunbanjo* \\ P. Arawomo** (iD) orcid.org/0000-0003-0814-0342
}

*University of Ibadan, Dept. of Mathematics, Ibadan, Nigeria. $\square$ ogunbanjoadeyemi@gmail.com

*University of Ibadan, Dept. of Mathematics, Ibadan, Nigeria. $\nabla$ womopeter@gmail.com

\begin{abstract}
:
By using averaging functions, we present some new oscillation criteria for the solution of a generalized forced nonlinear conformable fractional differential equation. The results obtained here extend and improve on some existing results. Examples are also given to show the validity of our results.
\end{abstract}

Keywords: Oscillation; Forced; Nonlinear conformable fractional differential equation.

MSC (2010): 34A08, 34A34, 34C15, 34D10.

Cite this article as (IEEE citation style):

A. Ogunbanjo and P. Arawomo, "Oscillation of solutions to a generalized forced nonlinear conformable fractional differential equation", Proyecciones (Antofagasta, On line), vol. 38, no. 3, pp. 429-445, Aug. 2019, doi: 10.22199/issn. 07176279-2019-03-0028. [Accessed dd-mm-yyyy].

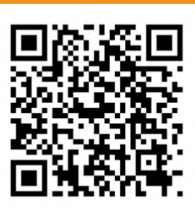

Article copyright: (C) 2019 Adeyemi Ogunbanjo and Peter Arawomo. This is an open access article distributed under the terms of the Creative Commons Licence, which permits unrestricted use and distribution provided the original author and source are credited. 


\section{Introduction}

In recent years there had been an increasing interest in fractional calculus because of its many applications in Science and Engineering see [5, 6, 9, 13] and references therein. Several researchers have worked on the oscillation of second order dynamic, sublinear and superlinear differential equations but not many have worked on oscillation of factional differential equations and the few have used Caputo, Riemann-Liouville and Modified RiemannLiouville such fractional derivatives see $[3,11,12,14,15]$. To the best of our knowledge only Jessada Tariboom and Sotiris K. Ntouyas [7] have worked on the oscillation of conformable fractional differential equations.

In this article, with the definition of conformable fractional derivative given by R. Khalil [8], we consider the establishment of oscillation of solutions to the generalized forced nonlinear conformable fractional differential equation

$T_{\alpha}\left[a(t) \psi(x(t)) T_{\alpha} x(t)\right]+P\left(t, x(t), T_{\alpha} x(t)\right)=Q\left(t, x(t), T_{\alpha} x(t)\right) t \geq t_{0}>0$,

$$
\alpha \in(1,2)
$$

where $T_{\alpha}($.$) denotes the operator called conformable fractional derivative$ of order $\alpha$ with respect to variable $t, C^{\alpha}$ denotes continuous function with fractional derivative of order $\alpha, a \in C^{\alpha}\left[\left[t_{0}, \infty\right), \mathbf{R}\right]$ and $P, Q \in C^{\alpha}\left[\left[t_{0}, \infty\right) \times\right.$ $\left.\mathbf{R}^{2}, \mathbf{R}\right]$.

\section{Preliminaries}

For the purpose of this paper, we state the following definitions and theorems without proof.

Definition 2.1. [8]

Given a function $f:[0, \infty) \rightarrow \mathbf{R}$. Then the "conformable fractional derivative" of $f$ of order $\alpha$ is defined by

$$
T_{\alpha}(f)(t)=\lim _{\epsilon \rightarrow 0} \frac{f\left(t+\epsilon t^{1-\alpha}\right)-f(t)}{\epsilon} \quad \forall t>0, \alpha \in(0,1)
$$

If $f$ is $\alpha$-differentiable in some $(0, a), a>0$, and $\lim _{t \rightarrow 0^{+}} f^{\alpha}(t)$ exists, then define

$$
f^{\alpha}(0)=\lim _{t \rightarrow 0^{+}} f^{\alpha}(t)
$$




\section{Definition 2.2. [8]}

Let $\alpha \in(n, n+1]$, and $f$ be an n-differentiable at $t$, where $t>0$. Then the conformable fractional derivative of $f$ of order $\alpha$ is defined as

$$
T_{\alpha}(f)(t)=\lim _{\epsilon \rightarrow 0} \frac{f^{\left.\left(\Gamma_{\alpha}\right\rceil-1\right)}\left(t+\epsilon t^{\left.\left(\Gamma_{\alpha}\right\rceil-\alpha\right)}\right)-f^{\left({ }^{(\alpha\rceil}-1\right)}(t)}{\epsilon} \forall t>0, \alpha \in(0,1)
$$

where $\alpha$ is the smallest integer greater than or equal to $\alpha$.

Definition 2.3. [8]

Let $\alpha \in(0,1]$ and $0 \leq a<b$. A function $f:[a, b] \rightarrow \mathbf{R}$ is $\alpha$-fractional integrable on $[a, b]$ if the integral

$$
\int_{a}^{b} f(x) d_{\alpha} x=\int_{a}^{b} f(x) x^{\alpha-1} d x
$$

exists and is finite. All $\alpha$-fractional integrable function on $[a, b]$ is denoted by $L_{\alpha}^{1}([a, b])$

We refer the readers who are not familiar with the properties of conformable fractional derivatives to the article of R. Khalil et-al [8] for clarification.

\section{Definition 2.4.}

The point $t_{0}$ is said to be a zero of $x(t)$ if $x\left(t_{0}\right)=0$.

\section{Definition 2.5.}

A solution $x(t)$ of (1.1) is said to be oscillatory if it has arbitrarily large zeros, otherwise it is said to be nonoscillatory. The equation is said to be oscillatory if all its solutions are oscillatory.

Theorem 2.6. $\{$ Integration by parts $[1]\}$

Let $f, g:[a, b] \rightarrow \mathbf{R}$ be two functions such that $f g$ is differentiable. Then

$$
\int_{a}^{b} f(x) T_{\alpha}^{a}(g)(x) d_{\alpha} x=\left.f g\right|_{a} ^{b}-\int_{a}^{b} g(x) T_{\alpha}^{a}(f)(x) d_{\alpha} x
$$

where $T($.$) represent the conformable fractional derivative of order \alpha$ 
Theorem 2.7. (ChainRule[1], [16])

Suppose $f, g:(a, \infty) \rightarrow \mathbf{R}$ be (left) $\alpha$-differentiable functions, where $0<\alpha \leq 1$. Let $h(t)=f(g(t))$. Then $h(t)$ is left $\alpha$-differentiable and for all $t$ with $t \neq a$ and $g(t) \neq 0$ we have

$$
\left(T_{\alpha}^{a} h\right)(t)=\left(T_{\alpha}^{a} f\right)(g(t)) \cdot\left(T_{\alpha}^{a} g\right)(t) \cdot g(t)^{\alpha-1}
$$

If $t=a$, we have

$$
\left(T_{\alpha}^{a} h\right)(a)=\lim _{t \rightarrow a^{+}}\left(T_{\alpha}^{a} f\right)(g(t)) \cdot\left(T_{\alpha}^{a} g\right)(t) \cdot g(t)^{\alpha-1}
$$

\section{Main Results}

In this section, we establish sufficient conditions for equation (1.1) to be oscillatory. We also introduce some functions $h, H \in C\left(\left[t_{0}, \infty\right), \mathbf{R}\right)$ satisfying $H(t, t)=0, H(t, s)>0, t>s \geq t_{0}$ with $H$ having continuous partial derivative $\frac{\partial H(t, s)}{\partial t}$ and $\frac{\partial H(t, s)}{\partial s}$ on $\left[t_{0}, \infty\right)$ such that

$$
\begin{aligned}
& \frac{\partial H(t, s)}{\partial t}=-h_{1}(t, s) \sqrt{H}(t, s) \\
& \frac{\partial H(t, s)}{\partial s}=-h_{2}(t, s) \sqrt{H}(t, s)
\end{aligned}
$$

Theorem 3.1. Assume that:

$\beta_{1}: x f(x)>0, \quad x \neq 0$

$\beta_{2}: f^{\prime}(x) \geq \mu>0, \quad x \neq 0$

$\beta_{3}: 0<\psi(x) \leq M$

$\beta_{4}: \frac{P\left(t, x, T_{\alpha} x(t)\right)}{f(x)} \geq p(t)$ and $\quad \frac{Q\left(t, x, T_{\alpha} x(t)\right)}{f(x)} \leq q(t)$ for $x \neq 0$

Also, suppose $\exists \varrho(t)$ and $g(t) \in C^{\alpha}\left[\left[t_{0}, \infty\right),(0, \infty)\right]$ such that

(3.1) $\limsup _{t \rightarrow \infty} \frac{1}{H\left(t, t_{0}\right)} \int_{t_{0}}^{t} \varrho(s)\left[\frac{H(t, s) \Phi(s)}{s^{1-\alpha}}-\frac{s^{1-\alpha} a(s) M}{4 \mu} h_{1}^{2}(t, s)\right] d s=\infty$

where

$$
\begin{gathered}
\varrho(s)=\exp \left(-2 \mu \int^{s} g(v) d v\right) \\
\Phi(t)=a(t) M \mu g^{2}(t)+p(t)-q(t)-T_{\alpha}[a(t) \psi(x(t)) g(t)]
\end{gathered}
$$

then every solution of (1.1) is oscillatory. 
Proof. Let $x(t)$ be a nonoscillatory solution of (1.1). Without loss of generality, we assume that $x(t)>0$ on $\left[\tau_{0}, \infty\right)$ for some $\tau_{0} \geq t_{0}$.

Define

$$
\begin{aligned}
u(t) & =\varrho(t)\left[\frac{a(t) \psi(x(t)) T_{\alpha} x(t)}{f(x(t))}+a(t) \psi(x(t)) g(t)\right] \\
T_{\alpha} u(t) & =\varrho(t) T_{\alpha}\left[\frac{a(t) \psi(x(t)) T_{\alpha} x(t)}{f(x(t))}+a(t) \psi(x(t)) g(t)\right] \\
& +\left[\frac{a(t) \psi(x(t)) T_{\alpha} x(t)}{f(x(t))}+a(t) \psi(x(t)) g(t)\right] T_{\alpha} \varrho(t) \\
& =\frac{\varrho(t) T_{\alpha}\left[a(t) \psi(x(t)) T_{\alpha} x(t)\right]}{f(x(t))}-\frac{\varrho(t)\left[a(t) \psi(x(t)) x^{\prime 2} t^{2(1-\alpha)}\right] f^{\prime}(x(t))}{f^{2}(x(t))} \\
+ & (t) T_{\alpha}[a(t) \psi(x(t)) g(t)]+\left[\frac{a(t) \psi(x(t)) T_{\alpha} x(t)}{f(x(t))}+a(t) \psi(x(t)) g(t)\right] t^{1-\alpha} \varrho^{\prime}(t)
\end{aligned}
$$

Using $\beta_{1}-\beta_{4}$ and (3.2) in (3.4), we have

$$
T_{\alpha} u(t) \leq-\frac{u^{2} \mu}{a(t) \varrho(t) M}-\varrho(t) \Phi(t)
$$

for $t \geq \tau_{0}$. It follows that for all $t \geq \tau \geq \tau_{0}$, we multiply (3.5) through by $H(t, s)$ and integrate both sides w.r.t $d_{\alpha} s$ from $\tau$ to $t$

$$
\begin{aligned}
& I_{\alpha}\left[H(t, s) T_{\alpha} u(s)\right] \leq I_{\alpha}\left[-H(t, s) \frac{u^{2} \mu}{a(s) \varrho(s) M}-H(t, s) \varrho(s) \Phi(s)\right] \\
& \int_{\tau}^{t} H(t, s) s^{1-\alpha} u^{\prime}(s) d_{\alpha} s \leq \int_{\tau}^{t}-\left[\frac{u^{2} \mu H(t, s)}{a(s) \varrho(s) M}+\varrho(s) H(t, s) \Phi(s)\right] d_{\alpha} s \\
& \int_{\tau}^{t} \varrho(s) \frac{H(t, s) \Phi(s)}{s^{1-\alpha}} d s \leq-\int_{\tau}^{t} s^{1-\alpha} H(t, s) u^{\prime}(s) d_{\alpha} s-\int_{\tau}^{t} \frac{u^{2} \mu H(t, s)}{s^{1-\alpha} a(s) \varrho(s) M} d s
\end{aligned}
$$

Using Theorem 2.6 on the first integral at the right hand side of inequality (3.6) above we have 


$$
\begin{aligned}
-\int_{\tau}^{t} s^{1-\alpha} H(t, s) u^{\prime}(s) d_{\alpha} s & =-\left[\left.H(t, s) u(s)\right|_{\tau} ^{t}-\int_{\tau}^{t} \dot{H}(t, s) u(s) d s\right] \\
& =H(t, \tau) u(\tau)-\int_{\tau}^{t}\left[-\frac{\partial}{\partial s} H(t, s) u(s)\right] d s
\end{aligned}
$$

$$
=H(t, \tau) u(\tau)-\int_{\tau}^{t} h_{1}(t, s) \sqrt{H(t, s)} u(s) d s
$$

substitute (3.7) into (3.6) to get

$$
\begin{aligned}
\int_{\tau}^{t} \varrho(s) \frac{H(t, s) \Phi(s)}{s^{1-\alpha}} d s & \leq H(t, \tau) u(\tau)-\int_{\tau}^{t} h_{1}(t, s) \sqrt{H(t, s)} u(s) d s \\
& -\int_{\tau}^{t} \frac{u^{2} \mu H(t, s)}{s^{1-\alpha} a(s) \varrho(s) M} d s
\end{aligned}
$$

simplifying, we have

$$
\int_{\tau}^{t} \varrho(s)\left[\frac{H(t, s) \Phi(s)}{s^{1-\alpha}}-\frac{s^{1-\alpha} a(s) M}{4 \mu} h_{1}^{2}(t, s)\right] d s \leq H(t, \tau) u(\tau)
$$

This implies that for every $t \geq \tau_{0}$,

$$
\begin{aligned}
\int_{\tau_{0}}^{t} \varrho(s)\left[\frac{H(t, s) \Phi(s)}{s^{1-\alpha}}-\frac{s^{1-\alpha} a(s) M}{4 \mu} h_{1}^{2}(t, s)\right] d s & \leq H\left(t, \tau_{0}\right) u\left(\tau_{0}\right) \\
& \leq H\left(t, t_{0}\right)\left|u\left(\tau_{0}\right)\right|
\end{aligned}
$$

Therefore,

$$
\begin{aligned}
& \int_{t_{0}}^{t} \varrho(s)\left[\frac{H(t, s) \Phi(s)}{s^{1-\alpha}}-\frac{s^{1-\alpha} a(s) M}{4 \mu} h_{1}^{2}(t, s)\right] d s \\
& =\int_{t_{0}}^{\tau_{0}} \varrho(s)\left[\frac{H(t, s) \Phi(s)}{s^{1-\alpha}}-\frac{s^{1-\alpha} a(s) M}{4 \mu} h_{1}^{2}(t, s)\right] d s
\end{aligned}
$$




$$
\begin{gathered}
+\int_{\tau_{0}}^{t} \varrho(s)\left[\frac{H(t, s) \Phi(s)}{s^{1-\alpha}}-\frac{s^{1-\alpha} a(s) M}{4 \mu} h_{1}^{2}(t, s)\right] d s \\
\leq H\left(t, t_{0}\right) \int_{t_{0}}^{\tau_{0}}\left|\frac{\varrho(s) \Phi(s)}{s^{1-\alpha}}\right| d s+H\left(t, t_{0}\right)\left|u\left(\tau_{0}\right)\right| \\
=H\left(t, t_{0}\right)\left[\int_{t_{0}}^{\tau_{0}}\left|\frac{\varrho(s) \Phi(s)}{s^{1-\alpha}}\right| d s+\left|u\left(\tau_{0}\right)\right|\right] \\
\limsup _{t \rightarrow \infty} \frac{1}{H\left(t, t_{0}\right)} \int_{t_{0}}^{t} \varrho(s)\left[\frac{H(t, s) \Phi(s)}{s^{1-\alpha}}-\frac{s^{1-\alpha} a(s) M}{4 \mu} h_{1}^{2}(t, s)\right] d s \\
\leq \int_{t_{0}}^{\tau_{0}}\left|\frac{\varrho(s) \Phi(s)}{s^{1-\alpha}}\right| d s+\left|u\left(\tau_{0}\right)\right|<\infty
\end{gathered}
$$

which contradicts (3.1). The proof is complete.

Example 1. For $t \geq 2$, consider the nonlinear forced fractional differential equation

$T_{\alpha}\left[2(x(t)+5) T_{\alpha} x(t)\right]+\left[\frac{1}{2} t^{-5 / 2}+T_{\alpha}(t \exp (x))\right] x(t)=t^{-1 / 2} x(t) \sin t+\frac{x^{2}(t) T_{\alpha}(\cos x)}{t^{3}\left(x^{3}(t)+1\right)}$

We set

$$
\left\{\begin{array}{r}
f(x(t))=x(t), f^{\prime}(x(t)) \geq \mu=1, a(t)=2 \\
x(t)=t+1, x^{\prime}(t)=1 \\
\psi(x(t))=x+5 \geq 5=M, g(t)=t^{-5 / 4} \\
H(t, s)=(t-s)^{\lambda}, \lambda=2, \alpha=\frac{4}{3}, \varrho(t)=t^{\frac{3}{2}}, t_{0}=2
\end{array}\right.
$$

Using $\beta_{4}$ in (3.9), we deduce that

$$
\begin{gathered}
\frac{P\left(t, x(t), T_{\alpha} x(t)\right)}{f(x)}=\frac{1}{2} t^{-5 / 2}+\left(t^{2-\alpha}+t^{1-\alpha}\right) \exp (t+1) \\
\geq \frac{1}{2} t^{-5 / 2}+t^{2 / 3}+t^{-1 / 3}=p(t)
\end{gathered}
$$

and

$$
\begin{aligned}
\frac{Q\left(t, x(t), T_{\alpha} x(t)\right)}{f(x(t))} & =t^{-1 / 2} \sin t+\frac{1}{t^{3}}\left(\frac{-x^{2-\alpha} \sin x(t)}{x^{3}(t)+1}\right) \\
& \leq t^{-1 / 2} \sin t=q(t)
\end{aligned}
$$


Also

$$
\left\{\begin{array}{r}
T_{\alpha}[a(t) \psi(x(t)) g(t)]=T_{\alpha}\left[2(t+6) \times t^{-5 / 4}\right]=-\frac{1}{2} t^{-19 / 12}-15 t^{-31 / 12} \\
h_{1}^{2}(t, s)=\left[\lambda(t-s)^{\lambda / 2-1}\right]^{2}=\lambda^{2}(t-s)^{(\lambda-2)}
\end{array}\right.
$$

substitute (3.10) - (3.13) into LHS of (3.1), we have

$$
\begin{aligned}
\limsup _{t \rightarrow \infty} \frac{1}{(t-2)^{2}} \int_{2}^{t} & {\left[(t-s)^{2}\left(\frac{21}{2} s^{-2 / 3}+s^{5 / 2}+s^{9 / 6}-s^{4 / 3} \sin s+\frac{1}{2} s^{1 / 4}+\frac{15}{2} t^{-3 / 4}\right)\right] d s } \\
& -\limsup _{t \rightarrow \infty} \frac{1}{(t-2)^{2}} \int_{2}^{t} 10 s^{11 / 6} d s=\infty
\end{aligned}
$$

This shows that (3.1) is satisfied and thus, equation (3.9) is oscillatory.

Theorem 3.2. Assume that $\beta_{1}-\beta_{4}$ in Theorem 3.1 hold. Let $\lambda>1$ be a constant. Suppose (3.1) does not hold such that $\exists$ a function $g \in$ $C^{\alpha}\left[\left[t_{0}, \infty\right),(0, \infty)\right]$ satisfying

$$
\limsup _{t \rightarrow \infty} \frac{1}{t^{\lambda}} \int_{t_{0}}^{t}\left[\frac{(t-s)^{\lambda} \varrho(s) \Phi(s)}{s^{1-\alpha}}-\frac{\lambda^{2}}{4 \mu}(t-s)^{\lambda-2} \varrho(s) a(s) M s^{1-\alpha}\right] d s=\infty
$$

where $\varrho(s)$ and $\Phi(s)$ are the same as equations (3.2) and (3.3) respectively. Then, every solution of (1.1) is oscillatory.

Proof. Without loss of generality, we assume that $\exists$ a solution of (1.1) such that $x(t)>0$ on $\left[\tau_{0}, \infty\right)$ for some $\tau_{0} \geq t_{0}$. Define $u(t)$ as in Theorem 3.1, then we obtained (3.5). Multiply (3.5) through by $(t-s)^{\lambda}$ and integrate both sides w.r.t $d_{\alpha} s$ from $\tau$ to $t$

$$
\begin{aligned}
I_{\alpha}\left[(t-s)^{\lambda} T_{\alpha} u(s)\right] & \leq I_{\alpha}\left[-(t-s)^{\lambda} \frac{u^{2} \mu}{a(s) \varrho(s) M}-(t-s)^{\lambda} \varrho(s) \Phi(s)\right] \\
\int_{\tau}^{t}(t-s)^{\lambda} s^{1-\alpha} u^{\prime}(s) d_{\alpha} s & \leq \int_{\tau}^{t}-\left[\frac{(t-s)^{\lambda} u^{2} \mu}{a(s) \varrho(s) M}+\varrho(s)(t-s)^{\lambda} \Phi(s)\right] d_{\alpha} s \\
\int_{\tau}^{t} \varrho(s) \frac{(t-s)^{\lambda} \Phi(s)}{s^{1-\alpha}} d s & \leq-\int_{\tau}^{t} s^{1-\alpha}(t-s)^{\lambda} u^{\prime}(s) d_{\alpha} s \\
& -\int_{\tau}^{t} \frac{(t-s)^{\lambda} u^{2} \mu}{s^{1-\alpha} a(s) \varrho(s) M} d s
\end{aligned}
$$


Using Theorem 2.6 on the first integral at the right hand side of the above inequality, we have

$$
\begin{aligned}
\int_{\tau}^{t} \varrho(s) \frac{(t-s)^{\lambda} \Phi(s)}{s^{1-\alpha}} d s & \leq(t-\tau)^{\lambda} u(\tau)-\lambda \int_{\tau}^{t}(t-s)^{\lambda-1} u(s) d s \\
& -\int_{\tau}^{t} \frac{(t-s)^{\lambda} u^{2} \mu}{s^{1-\alpha} a(s) \varrho(s) M} d s \leq(t-\tau)^{\lambda} u(\tau) \\
& -\int_{\tau}^{t}\left[\frac{(t-s)^{\lambda} \mu}{\varrho(s) a(s) M s^{1-\alpha}} u^{2}(s)+\lambda(t-s)^{\lambda-1} u(s)\right] d s
\end{aligned}
$$

Therefore, for every $t \geq t_{0}$

$\begin{aligned} \limsup _{t \rightarrow \infty} \frac{1}{t^{\lambda}} \int_{t_{0}}^{t}\left[\varrho(s) \frac{(t-s)^{\lambda} \Phi(s)}{s^{1-\alpha}}-\frac{\lambda^{2}(t-s)^{\lambda-2} \varrho(s) a(s) M s^{1-\alpha}}{4 \mu}\right] d s & \leq u\left(t_{0}\right) \\ & <\infty\end{aligned}$

which contradicts (3.14). The proof is complete.

Theorem 3.3. For sufficiently large $\tau \geq t_{0}, \exists \eta_{2}, \eta_{1}$ and $\eta_{3}$ with $\tau \leq \eta_{2}<$ $\eta_{1}<\eta_{3}$. Assume that $\beta_{1}-\beta_{4}$ hold with (3.1)- (3.3) not holding. Also, if there exist $\varrho(t) \in C^{\alpha}\left[\left[t_{0}, \infty\right),(0, \infty)\right]$ such that

$$
\begin{aligned}
& \frac{1}{H\left(\eta_{3}, \eta_{1}\right)} \int_{\eta_{1}}^{\eta_{3}} H\left(\eta_{3}, s\right) \frac{\varrho(s)}{s^{1-\alpha}}[p(s)-q(s)] d s \\
+ & \frac{1}{H\left(\eta_{1}, \eta_{2}\right)} \int_{\eta_{2}}^{\eta_{1}} H\left(s, \eta_{2}\right) \frac{\varrho(s)}{s^{1-\alpha}}[p(s)-q(s)] d s \\
> & \frac{1}{4 H\left(\eta_{3}, \eta_{1}\right)} \int_{\eta_{1}}^{\eta_{3}} \frac{M \varrho(s) a(s) s^{1-\alpha}}{\mu} \chi_{2}^{2}\left(\eta_{3}, s\right) d s \\
+ & \frac{1}{4 H\left(\eta_{1}, \eta_{2}\right)} \int_{\eta_{2}}^{\eta_{1}} \frac{M \varrho(s) a(s) s^{1-\alpha}}{\mu} \chi_{1}^{2}\left(s, \eta_{2}\right) d s
\end{aligned}
$$

where

$$
\left\{\begin{array}{l}
\chi_{1}(t, s)=h_{1}(t, s)-\frac{\varrho^{\prime}(s)}{\varrho(s)} \sqrt{H(t, s)} \\
\chi_{2}(s, t)=h_{2}(s, t)-\frac{\varrho^{\prime}(s)}{\varrho(s)} \sqrt{H(s, t)}
\end{array}\right.
$$

then, every solution of equation (1.1) is oscillatory. 
Proof. Suppose the contrary, that is, $x(t)$ is a non-oscillatory solution of equation $(1.1)$ on $\left[\tau_{0}, \infty\right)$.

Define

$$
\begin{gathered}
u(t)=\varrho(t) \frac{a(t) \psi(x(t)) T_{\alpha} x(t)}{f(x(t))} \quad t \geq \tau_{0} \geq t_{0} \\
T_{\alpha} u(t)=T_{\alpha}\left[\varrho(t) \frac{a(t) \psi(x(t)) T_{\alpha} x(t)}{f(x(t))}\right]
\end{gathered}
$$

Then, by using $\beta_{1}-\beta_{4}$ in Theorem 3.1 on (3.17), we obtain

$(3.18) \varrho(t)[p(t)-q(t)] \leq-t^{1-\alpha} u^{\prime}(t)-\frac{\mu}{M \varrho(t) a(t)} u^{2}(t)+\frac{\varrho^{\prime}(t) t^{1-\alpha}}{\varrho(t)} u(t)$

Multiplying both sides of (3.18) by $H(t, s)$ and integrating with respect to $d_{\alpha} s$ from $\eta_{1}$ to $t$ for $t \in\left[\eta_{1}, \eta_{3}\right)$, we have

$$
\begin{aligned}
\int_{\eta_{1}}^{t} H(t, s) \frac{\varrho(s)}{s^{1-\alpha}}[p(s)-q(s)] d s & \leq-\int_{\eta_{1}}^{t} s^{1-\alpha} H(t, s) u^{\prime}(s) d_{\alpha} s \\
& -\int_{\eta_{1}}^{t} H(t, s) \frac{\mu}{M \varrho(s) a(s) s^{1-\alpha}} u^{2}(s) d s \\
& +\int_{\eta_{1}}^{t} H(t, s) \frac{\varrho^{\prime}(s)}{\varrho(s)} u(s) d s
\end{aligned}
$$

Using Theorem 2.6 on the first integral at the right hand side, we have

$$
\begin{aligned}
& \int_{\eta_{1}}^{t} H(t, s) \frac{\varrho(s)}{s^{1-\alpha}}[p(s)-q(s)] d s \leq H\left(t, \eta_{1}\right) u\left(\eta_{1}\right) \\
&-\int_{\eta_{1}}^{t} h_{1}(t, s) \sqrt{H(t, s)} u(s) d s \\
&-\int_{\eta_{1}}^{t} H(t, s) \frac{\mu}{M \varrho(s) a(s) s^{1-\alpha}} u^{2}(s) d s \\
&+\int_{\eta_{1}}^{t} H(t, s) \frac{\varrho^{\prime}(s)}{\varrho(s)} u(s) d s \\
& \leq H\left(t, \eta_{1}\right) u\left(\eta_{1}\right)+\int_{\eta_{1}}^{t} \frac{M \varrho(s) a(s) s^{1-\alpha}}{4 \mu} \chi_{1}^{2}(t, s) d s
\end{aligned}
$$

divide (3.19) by $H\left(t, \eta_{1}\right)$ and let $t \rightarrow \eta_{3}^{-}$, then we obtain

$$
\frac{1}{H\left(\eta_{3}, \eta_{1}\right)} \int_{\eta_{1}}^{\eta_{3}} H\left(\eta_{3}, s\right) \frac{\varrho(s)}{s^{1-\alpha}}[p(s)-q(s)] d s \leq u\left(\eta_{1}\right)
$$




$$
+\frac{1}{4 H\left(\eta_{3}, \eta_{1}\right)} \int_{\eta_{1}}^{\eta_{3}} \frac{M \varrho(s) a(s) s^{1-\alpha}}{\mu} \chi_{1}^{2}\left(\eta_{3}, s\right) d s
$$

In the same way, we multiply both sides of $(3.18)$ by $H(s, t)$ and integrate with respect to $d_{\alpha} s$ for $t \in\left(\eta_{2}, \eta_{1}\right]$ to get

$$
\begin{aligned}
\int_{t}^{\eta_{1}} H(s, t) \frac{\varrho(s)}{s^{1-\alpha}}[p(s)-q(s)] d s & \leq-\int_{t}^{\eta_{1}} s^{1-\alpha} H(s, t) u^{\prime}(s) d_{\alpha} s \\
& -\int_{t}^{\eta_{1}} H(s, t) \frac{\mu}{M \varrho(s) a(s) s^{1-\alpha}} u^{2}(s) \\
& +\int_{t}^{\eta_{1}} H(s, t) \frac{\varrho^{\prime}(s)}{\varrho(s)} u(s) d s
\end{aligned}
$$

Following the same process in (3.20) with $t \rightarrow \eta_{2}^{-}$, we arrive at

$$
\begin{gathered}
\int_{\eta_{2}}^{\eta_{1}} H\left(s, \eta_{2}\right) \frac{\varrho(s)}{s^{1-\alpha}}[p(s)-q(s)] d s \leq-u\left(\eta_{1}\right) \\
+\frac{1}{4 H\left(\eta_{1}, \eta_{2}\right)} \int_{\eta_{2}}^{\eta_{1}} \frac{M \varrho(s) a(s) s^{1-\alpha}}{\mu} \chi_{2}^{2}\left(s, \eta_{2}\right) d s
\end{gathered}
$$

Add (3.20) and (3.21) together to obtain

$$
\begin{aligned}
& \frac{1}{H\left(\eta_{3}, \eta_{1}\right)} \int_{\eta_{1}}^{\eta_{3}} H\left(\eta_{3}, s\right) \frac{\varrho(s)}{s^{1-\alpha}}[p(s)-q(s)] d s \\
+ & \frac{1}{H\left(\eta_{1}, \eta_{2}\right)} \int_{\eta_{2}}^{\eta_{1}} H\left(s, \eta_{2}\right) \frac{\varrho(s)}{s^{1-\alpha}}[p(s)-q(s)] d s \\
\leq & \frac{1}{4 H\left(\eta_{3}, \eta_{1}\right)} \int_{\eta_{1}}^{\eta_{3}} \frac{M \varrho(s) a(s) s^{1-\alpha}}{\mu} \chi_{1}^{2}\left(\eta_{3}, s\right) d s \\
+ & \frac{1}{4 H\left(\eta_{1}, \eta_{2}\right)} \int_{\eta_{2}}^{\eta_{1}} \frac{M \varrho(s) a(s) s^{1-\alpha}}{\mu} \chi_{2}^{2}\left(s, \eta_{2}\right) d s
\end{aligned}
$$

which contradicts (3.15). The proof is thus complete.

Theorem 3.4. Under the conditions of Theorem 3.3, Suppose (3.15) does not hold such that

$$
\begin{gathered}
\frac{1}{\left(\eta_{3}-\eta_{1}\right)^{\lambda}} \int_{\eta_{1}}^{\eta_{3}}\left(\eta_{3}-s\right)^{\lambda} \frac{\varrho(s)}{s^{1-\alpha}}[p(s)-q(s)] d s \\
+\frac{1}{\left(\eta_{1}-\eta_{2}\right)^{\lambda}} \int_{\eta_{2}}^{\eta_{1}}\left(s-\eta_{2}\right)^{\lambda} \frac{\varrho(s)}{s^{1-\alpha}}[p(s)-q(s)] d s
\end{gathered}
$$




$$
\begin{aligned}
& >\frac{1}{4\left(\eta_{3}-\eta_{1}\right)^{\lambda}} \int_{\eta_{1}}^{\eta_{3}} \frac{M \varrho(s) a(s) s^{1-\alpha}}{\mu}\left(\eta_{3}-s\right)^{\lambda-2}\left(\lambda-\frac{\varrho^{\prime}(s)}{\varrho(s)}\left(\eta_{3}-s\right)\right)^{2} d s \\
& +\frac{1}{4\left(\eta_{1}-\eta_{2}\right)^{\lambda}} \int_{\eta_{2}}^{\eta_{1}} \frac{M \varrho(s) a(s) s^{1-\alpha}}{\mu}\left(s-\eta_{2}\right)^{\lambda-2}\left(\lambda+\frac{\varrho^{\prime}(s)}{\varrho(s)}\left(s-\eta_{2}\right)\right)^{2} d s
\end{aligned}
$$

then, equation (1.1) is oscillatory.

Proof. Let $x(t)$ be a non-oscillatory solution of (1.1). Following the proof of Theorem 3.3, we obtain (3.18). Multiply (3.18) by $(t-s)^{\lambda}$ and integrate with respect to $d_{\alpha} s$ from $\eta_{1}$ to $t$ for $t \in\left[\eta_{1}, \eta_{3}\right)$ so that

$$
\begin{gathered}
\int_{\eta_{1}}^{t}(t-s)^{\lambda} \frac{\varrho(s)}{s^{1-\alpha}}[p(s)-q(s)] d s \leq-\int_{\eta_{1}}^{t}(t-s)^{\lambda} u^{\prime}(s) d s \\
(3.23)-\int_{\eta_{1}}^{t}(t-s)^{\lambda} \frac{\mu}{M \varrho(s) a(s) s^{1-\alpha}} u^{2}(s) d s+\int_{\eta_{1}}^{t}(t-s)^{\lambda} \frac{\varrho^{\prime}(s)}{\varrho(s)} u(s) d s
\end{gathered}
$$

By Theorem 2.6, (3.23) becomes

$$
\begin{aligned}
& \int_{\eta_{1}}^{t}(t-s)^{\lambda} \frac{\varrho(s)}{s^{1-\alpha}}[p(s)-q(s)] d s \leq\left(t-\eta_{1}\right)^{\lambda} u\left(\eta_{1}\right)-\int_{\eta_{1}}^{t} \lambda(t-s)^{\lambda-1} u(s) d s \\
&-\int_{\eta_{1}}^{t}(t-s)^{\lambda} \frac{\mu}{M \varrho(s) a(s) s^{1-\alpha}} u^{2}(s) d s \\
&+\int_{\eta_{1}}^{t}(t-s)^{\lambda} \frac{\varrho^{\prime}(s)}{\varrho(s)} u(s) d s \\
&=\left(t-\eta_{1}\right)^{\lambda} u\left(\eta_{1}\right) \\
&-\int_{\eta_{1}}^{t}\left[(t-s)^{\lambda} \frac{\mu}{M \varrho(s) a(s) s^{1-\alpha}} u^{2}(s)\right. \\
&\left.+(t-s)^{\lambda-1}\left[\lambda-(t-s) \frac{\varrho^{\prime}(s)}{\varrho(s)}\right] u(s)\right] d s \\
& \leq\left(t-\eta_{1}\right)^{\lambda} u\left(\eta_{1}\right)+\frac{1}{4} \int_{\eta_{1}}^{t} \frac{M \varrho(s) a(s) s^{1-\alpha}}{\mu}(t-s)^{\lambda-2}\left[\lambda-(t-s) \frac{\varrho^{\prime}(s)}{\varrho(s)}\right]^{2} d s
\end{aligned}
$$


Letting $t \rightarrow \eta_{3}^{-}$in (3.24) and dividing the result by $\left(\eta_{3}-\eta_{1}\right)^{\lambda}$, we have

$$
\begin{gathered}
\frac{1}{\left(\eta_{3}-\eta_{1}\right)^{\lambda}} \int_{\eta_{1}}^{\eta_{3}}\left(\eta_{3}-s\right)^{\lambda} \frac{\varrho(s)}{s^{1-\alpha}}[p(s)-q(s)] d s \leq u\left(\eta_{1}\right) \\
+\frac{1}{4 \mu\left(\eta_{3}-\eta_{1}\right)^{\lambda}} \int_{\eta_{1}}^{\eta_{3}} M \varrho(s) a(s) s^{1-\alpha}\left(\eta_{3}-s\right)^{\lambda-2}\left[\lambda-\left(\eta_{3}-s\right) \frac{\varrho^{\prime}(s)}{\varrho(s)}\right]^{2} d s
\end{gathered}
$$

Following the same process as above, multiplying both sides of (3.18) by $(s-t)^{\lambda}$ and then integrating with respect to $d_{\alpha} s$ from $t$ to $\eta_{1}$ for $t \in\left[\eta_{2}, \eta_{1}\right)$, we have

$$
\begin{gathered}
\int_{t}^{\eta_{1}}(s-t)^{\lambda} \frac{\varrho(s)}{s^{1-\alpha}}[p(s)-q(s)] d s \leq-\left(\eta_{1}-t\right)^{\lambda} u\left(\eta_{1}\right) \\
+\frac{1}{4} \int_{t}^{\eta_{1}} \frac{M \varrho(s) a(s) s^{1-\alpha}}{\mu}(s-t)^{\lambda-2}\left[\lambda+\left(s-\eta_{2}\right) \frac{\varrho^{\prime}(s)}{\varrho(s)}\right]^{2} d s
\end{gathered}
$$

Letting $t \rightarrow \eta_{2}^{-}$and dividing through by $\left(\eta_{1}-\eta_{2}\right)^{\lambda}$, we have

$$
\begin{gathered}
\frac{1}{\left(\eta_{1}-\eta_{2}\right)^{\lambda}} \int_{\eta_{2}}^{\eta_{1}}\left(s-\eta_{2}\right)^{\lambda} \frac{\varrho(s)}{s^{1-\alpha}}[p(s)-q(s)] d s \leq-u\left(\eta_{1}\right) \\
+\frac{1}{4 \mu\left(\eta_{1}-\eta_{2}\right)^{\lambda}} \int_{\eta_{2}}^{\eta_{1}} M \varrho(s) a(s) s^{1-\alpha}\left(s-\eta_{2}\right)^{\lambda-2}\left[\lambda+\left(s-\eta_{2}\right) \frac{\varrho^{\prime}(s)}{\varrho(s)}\right]^{2} d s
\end{gathered}
$$

Adding (3.25) and (3.26) together we have

$$
\begin{gathered}
\frac{1}{\left(\eta_{3}-\eta_{1}\right)^{\lambda}} \int_{\eta_{1}}^{\eta_{3}}\left(\eta_{3}-s\right)^{\lambda} \frac{\varrho(s)}{s^{1-\alpha}}[p(s)-q(s)] d s+ \\
\frac{1}{\left(\eta_{1}-\eta_{2}\right)^{\lambda}} \int_{\eta_{2}}^{\eta_{1}}\left(s-\eta_{2}\right)^{\lambda} \frac{\varrho(s)}{s^{1-\alpha}}[p(s)-q(s)] d s \\
\leq \frac{1}{4 \mu\left(\eta_{3}-\eta_{1}\right)^{\lambda}} \int_{\eta_{1}}^{\eta_{3}} M \varrho(s) a(s) s^{1-\alpha}\left(\eta_{3}-s\right)^{\lambda-2}\left[\lambda-\left(\eta_{3}-s\right) \frac{\varrho^{\prime}(s)}{\varrho(s)}\right]^{2} d s
\end{gathered}
$$




$$
+\frac{1}{4 \mu\left(\eta_{1}-\eta_{2}\right)^{\lambda}} \int_{\eta_{2}}^{\eta_{1}} M \varrho(s) a(s) s^{1-\alpha}\left(s-\eta_{2}\right)^{\lambda-2}\left[\lambda+\left(s-\eta_{2}\right) \frac{\varrho^{\prime}(s)}{\varrho(s)}\right]^{2} d s
$$

which contradicts (3.22). This completes the proof.

Example 2. For $t \geq 2$, consider the nonlinear forced fractional differential equation

$$
\begin{gathered}
T_{\alpha}\left[2\left(x^{2}(t)+3\right) T_{\alpha} x(t)\right]+t^{4} \frac{x(t)}{8}\left[4 t^{1 / 2}+\exp \left(\frac{1}{\alpha} t^{\alpha}\right)-2 t^{-2 \alpha} T_{\alpha} x(t)\right] \\
=t^{-3 / 2} x(t)+x^{2}(t) T_{\alpha}\left(\cos \frac{1}{\alpha} x(t)\right)
\end{gathered}
$$

We set

$$
\left\{\begin{array}{r}
f(x)=x(t), f^{\prime}(x)=1=\mu, a(t)=2 \\
x=t+1, x^{\prime}(t)=1 \\
\psi(x)=x^{2}+3 \geq 3=M, g(t)=t^{-5 / 4} \\
\alpha=\frac{4}{3}, \varrho(t)=t^{3 / 2}, t_{0}=2 \\
\eta_{1}=4, \eta_{2}=2, \eta_{3}=5
\end{array}\right.
$$

Using $\beta_{4}$ in (3.27), we deduce that

$$
\begin{aligned}
\frac{P\left(t, x(t), T_{\alpha} x(t)\right)}{f(x)} & =\frac{t^{4}}{8}\left[4 t^{1 / 2}+\exp \left(\frac{1}{\alpha} t^{\alpha}\right)-2 t^{-2 \alpha} T_{\alpha} x(t)\right] \\
& =\frac{t^{9 / 2}}{2}+\frac{t^{4}}{8} \exp \left(\frac{1}{\alpha} t^{\alpha}\right)-\frac{t^{5-3 \alpha}}{4} x^{\prime}(t) \\
& \geq \frac{t^{9 / 2}}{2}-\frac{t^{5-3 \alpha}}{4} x^{\prime}(t)=\frac{t^{9 / 2}}{2}-\frac{t}{4}=p(t) \\
\frac{Q\left(t, x(t), T_{\alpha} x(t)\right)}{f(x(t))} & =t^{-3 / 2}-\frac{x^{2-\alpha}(t)}{\alpha} \sin \frac{1}{\alpha} x(t) \\
& \leq t^{-3 / 2}=q(t)
\end{aligned}
$$

Also note that

$$
\frac{\varrho(t)}{t^{1-\alpha}}[p(t)-q(t)]=\frac{t^{19 / 3}}{2}-\frac{t^{17 / 6}}{2}-t^{1 / 3}
$$

substitute $p(t), q(t),(3.28)$ and (3.29) into (3.15), we have 


$$
\begin{aligned}
L H S & =\frac{1}{H\left(\eta_{1}, \eta_{2}\right)} \int_{\eta_{2}}^{\eta_{1}} \frac{H\left(s, \eta_{2}\right)}{s^{1-\alpha}} \varrho(s)[p(s)-q(s)] d s \\
& +\frac{1}{H\left(\eta_{3}, \eta_{1}\right)} \int_{\eta_{1}}^{\eta_{3}} \frac{H\left(\eta_{3}, s\right)}{s^{1-\alpha}} \varrho(s)[p(s)-q(s)] d s \\
& =\frac{1}{(5-4)^{2}} \int_{4}^{5}(5-s)^{2}\left[\frac{s^{19 / 3}}{2}-\frac{s^{17 / 6}}{2}-s^{1 / 3}\right] d s \\
& +\frac{1}{(4-2)^{2}} \int_{2}^{4}(s-2)^{2}\left[\frac{s^{19 / 3}}{2}-\frac{s^{17 / 6}}{2}-s^{1 / 3}\right] d s \\
& =1644.7+274.3=1919
\end{aligned}
$$

Similarly

$$
\begin{aligned}
R H S & =\frac{1}{4 H\left(\eta_{1}, \eta_{2}\right)} \int_{\eta_{2}}^{\eta_{1}} \frac{\varrho(s) a(s) M}{\mu} \chi_{2}^{2}\left(s, \eta_{2}\right) d s \\
& +\frac{1}{4 H\left(\eta_{3}, \eta_{1}\right)} \int_{\eta_{1}}^{\eta_{3}} \frac{\varrho(s) a(s) M}{\mu} \chi_{1}^{2}\left(\eta_{3}, s\right) d s \\
& =\frac{1}{4} \int_{4}^{5} 6 s^{7 / 6}\left[2-\frac{3}{2 s}(5-s)\right]^{2} d s \\
& +\frac{1}{16} \int_{2}^{4} 6 s^{7 / 6}\left[2-\frac{3}{2 s}(s-2)\right]^{2} d s \\
& =29.3+6.125=35.4
\end{aligned}
$$

Since the LHS > RHS, equation (3.15) is satisfied, whence (3.27) is oscillatory.

\section{References}

[1] T. Abdeljawad, "On conformable fractional calculus", Journal of Computational and Applied Mathematics, vol. 279, pp. 57-66, 2015, doi: 10.1016/j.cam.2014.10.016.

[2] F. Zulfeqarr, A. Ujlayan, and P. Ahuja, "A new fractional derivative and its fractional integral with some example”, Apr. 2017. arXiv:1705.00962. 
[3] Q. Feng, "Interval Oscillation Criteria for a Class of Nonlinear Fractional Differential Equations with Nonlinear Damping Term", IAENG International Journal of Applied Mathematics, vol. 43, no. 3, pp. 154-159, Aug. 2013. [On line]. Available: http://bit.ly/31iDarg

[4] F. Usta and M. Sarıkaya, "Explicit bounds on certain integral inequalities via conformable fractional calculus", Cogent Mathematics, vol. 4, no. 1, 2017, doi: 10.1080/23311835.2016.1277505.

[5] S. Grace and B. Lalli, "Oscillation theorems for second order superlinear differential equations with damping", Journal of the Australian Mathematical Society. Series A. Pure Mathematics and Statistics, vol. 53, no. 2, pp. 156-165, Oct. 1992, doi: 10.1017/s144678870003576x.

[6] J. Wong, "An oscillation theorem for second order sublinear differential equations", Proceedings of the American Mathematical Society, vol. 110 , no. 3, pp. 633-633, 1990, doi: 10.1090/S0002-9939-19901000170-4.

[7] J. Tariboon and S. Ntouyas, "Oscillation of impulsive conformable fractional differential equations", Open Mathematics, vol. 14, no. 1, 2016, doi: 10.1515/math-2016-0044.

[8] R. Khalil, M. Horani, A. Yousef, and M. Sababheh, "A new definition of fractional derivative", Journal of Computational and Applied Mathematics, vol. 264, pp. 65-70, 2014, doi: 10.1016/j.cam.2014.01.002.

[9] M. Kwong and J. Wong, "An application of integral inequality to second order nonlinear oscillation”, Journal of Differential Equations, vol. 46, no. 1, pp. 63-77, 1982, doi: 10.1016/0022-0396(82)90110-3.

[10] M. Sarikaya and F. Usta; "On Comparison Theorems for Conformable Fractional Differential Equations", International Journal of Analysis and Applications, vol. 12, no. 2, pp. 207-214, 2016. [On line]. Available: http://bit.ly/2yzvdld

[11] M. Remili, "Oscillation criteria for second order nonlinear perturbed differential equations", Electronic Journal of Qualitative Theory of Differential Equations, no. 25, pp. 1-11, May 2010, doi: 10.14232/ejqtde.2010.1.25.

[12] Q. Feng and F. Meng, "Oscillation of Solutions to Nonlinear forced fractional differential equations", Electronic Journal of Differential Equations, vol. 2013, no. 169, pp. 1-10, Jul. 2013. [On line]. Available: http://bit.ly/2MBeuq4

[13] R. Agarwal, M. Bohner, and W. Li, Nonoscillation and oscillation: theory for functional differential equations. New York: Marcel Dekker, 2004. 
[14] S. Grace, R. Agarwal, P. Wong, and A. Zafer, "On the oscillation of fractional differential equations", Fractional Calculus and Applied Analysis, vol. 15, no. 2, Mar. 2012, doi: 10.2478/s13540-012-0016-1.

[15] S. Öğrekçi, "Interval oscillation criteria for functional differential equations of fractional order", Advances in Difference Equations, vol. 2015, no. 1, Jan. 2015, doi: 10.1186/s13662-014-0336-z.

[16] Y. Çenesiz and A. Kurt, "The solutions of time and space conformable fractional heat equations with conformable Fourier transform", Acta Universitatis Sapientiae, Mathematica, vol. 7, no. 2, pp. 130-140, Feb. 2015, doi: 10.1515/ausm-2015-0009. 